\title{
VALORISATION DU ZOOPLANCTON PRODUIT EN ÉTANGS DE LAGUNAGE COMME BASE POUR L'ALIMENTATION DE LARVES ET JUVÉNILES DE CYPRINIDÉS
}

\author{
F. GUERRIN ( $)$ \\ CEMAGREF - Aménagements littoraux et Aquaculture \\ B.P. 5095 - 34033 MONTPELLIER Cedex (France)
}

(") adresse actuelle : INRA station de biométrie et d'intelligence artificielle

B.P. 27 - 31326 CASTANET-TOLOSAN Cedex (France)

Reçu le 6 octobre 1988

Accepté le 16 décembre 1988

Received 6 October, 1988

Accepted 16 December, 1988

\section{RÉSUME}

Quelques données chiffrées concernant la production zooplanctonique des étangs de lagunage d'eaux usées sont présentées :

- caractéristiques physico-chimiques du milieu,

- fluctuations d'abondance et évaluation de la biomasse récoltable.

Ces indications peuvent, dans une certaine mesure, constituer des repères pour une production de proies zooplanctoniques dans d'autres conditions.

La composition en protéines et en acides aminés de cette biomasse est discutée en référence aux besoins alimentaires connus pour quelques espèces de poissons dont la carpe (Cyprinus carpio). Un test de croissance à partir d'un aliment expérimental ayant comme unique base protéique du zooplancton déshydraté permet d'avancer un taux de substitution possible de $40 \%$ de protéines conventionnelles.

Un essai d'élevage larvaire de carpes ( 0 à 4 semaines) alimentées avec du zooplancton vivant a permis d'obtenir des résultats de survie de 70 et $99 \%$ selon la densité de larves introduites et la quantité de proies distribuées, pour des poids moyens respectivement de 1,95 et $0,67 \mathrm{~g}$ en fin d'élevage.

\section{VALORIZATION OF WASTE WATER TREATMENT PONDS ZOOPLANKTON AS A BASIS TO FEED LARVAE AND JUVENILES OF CYPRINIDS}

\section{ABSTRACT} given :

Some data concerning zooplankton production in domestic sewage treatment ponds are

- physico-chemical characteristics of the water.

- abundance fluctuation and harvestable biomass estimation.

These values can constitute a basis for zooplanktonic prey production in other conditions.

The protein and amino-acid composition of the biomass is discussed with reference to established nutritionnal needs of some fish species, with emphasis on the common carp (Cyprinus carpio). A growing test with experimental pellets having as unique protein source dried zooplankton, allowed us to assess a possible replacement rate of $40 \%$ of conventional proteins.

A rearing trial of mirror carp larvae ( 0 to 4 weeks) fed with live zooplankton resulted in a survival rate of $70 \%$ and $99 \%$, depending on the larvae and zooplankton densities, with an average individual weight of respectively 1.95 and 0.67 grams at the end of the experiment.

\section{INTRODUCTION}

Jusqu'à présent, l'utilisation de proies vivantes zooplanctoniques était considérée comme obligatoire dans la production de masse de juvéniles de presque toutes les espèces de poissons à l'exception de la truite (TAMAS et HORVATH, 1976).

Les résultats très encourageants acquis depuis quelques années dans la mise au point d'aliments inertes (CHARLON et BERGOT, 1984) peuvent, à plus ou moins long terme, déboucher sur une simplification considérable des procédures d'élevage. 
Dans cette attente, il a néanmoins paru intéressant de rassembler les données pratiques de valorisation du zooplancton collecté en étangs soumis à une fertilisation organique poussée, comme c'est le cas des lagunes d'épuration d'eaux usées domestiques.

Nous examinerons successivement les trois aspects qui, à notre avis, conditionnent la valorisation de cette ressource en aquaculture:

- l'estimation de la production récoltable : l'hypereutrophisation provoquée dans ces étangs, en réduisant la diversité spécifique, permet de générer de très fortes biomasses zooplanctoniques dont il importe de cerner les fluctuations quantitatives et qualitatives;

- la valeur alimentaire de la biomasse récoltée au regard des exigences des poissons: sa forte teneur en protéines et la qualité de sa composition en acides aminés nous ont amené à envisager son utilisation comme base protéique d'aliments composés;

- son utilisation sous forme de proies vivantes pour l'élevage larvaire: ces méthodes offrent une alternative acceptable aux cultures contrólées (Brachionus, Artemia) dont le coût élevé est un handicap majeur (BARNABÉ, 1979).

\section{MATÉRIEL ET MÉTHODES}

\section{Production de zooplancton en étangs de lagunage naturel}

L'étude a porté principalement sur le lagunage de la commune de Réalmont (Tarn), qui comporte une série de trois bassins de superficies respectives: $11.000 \mathrm{~m}^{2}, 9.000 \mathrm{~m}^{2}$ et $6.000 \mathrm{~m}^{2}$ (fig. 1). Dix-neuf campagnes de 24 heures réparties sur un cycle annuel ont été effectuées permettant:

- des mesures physico-chimiques réalisées in situ: température, oxygène dissous, $\mathrm{pH}$, transparence (disque de Secchi);

- la filtration totale du rejet de chacune des trois lagunes à l'aide de manches filtrantes de vide de maille $250 \mu \mathrm{m}$ relevées toutes les deux heures. Le zooplancton récolté à l'issue de chacune de ces périodes horaires était égoutté puis pesé (poids frais) et deux échantillons prélevés: l'un formolé, destiné à l'identification des formes présentes, le second, conservé au froid, afin de pratiquer la détermination du poids sec et les analyses nécessaires à l'étude de la valeur alimentaire (cf.2.1.).

Lors d'une précédente étude (GUERRIN, 1981), des analyses d'eau avaient également été réalisées au cours de 6 campagnes (matières en suspension, DB05, DCO, formes de l'azote, ortho-phosphates).

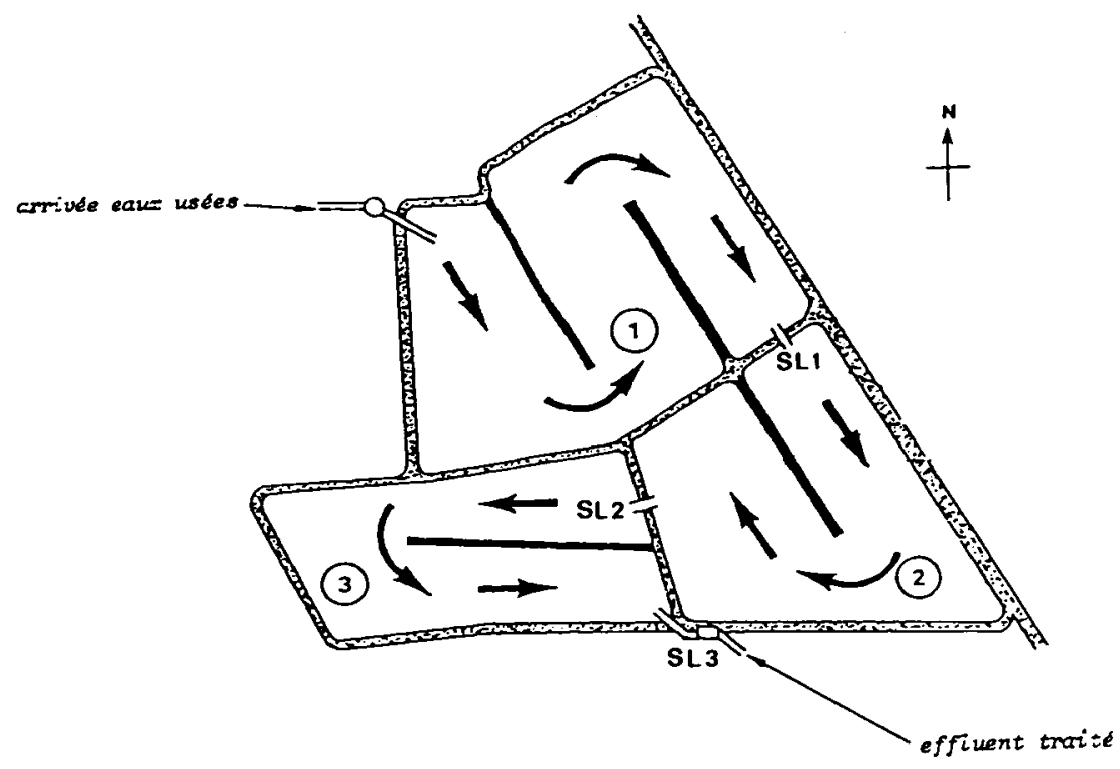

figure 1: $\quad$ Plan schématique du lagunage de Réalmont (Tarn). Profondeurs : L1 $=1,70 \mathrm{~m}$; $\mathbf{L 2}=1,30 \mathrm{~m} ; \mathrm{L3}=1,10 \mathrm{~m}$.

figure 1: Realmont (Tarn, France) domestic sewage treatment pond. Pond depths : $L_{1}=1.70 \mathrm{~m}, \mathrm{~L} 2=1.30 \mathrm{~m}, \mathrm{L3}=1.10 \mathrm{~m}$. 


\section{Valeur alimentaire du zooplancton}

\subsection{Approche analytique}

Les analyses ont porté sur la détermination de la matière sèche (séchage à l'étuve à $105^{\circ} \mathrm{C}$ jusqu'à poids constant), des cendres (calcination à $550^{\circ} \mathrm{C}$ ), de l'azote Kjeldahl permettant l'estimation de la teneur en protéines brutes $\left(N^{*} 6,25\right)$, et des acides aminés (lyophilisation des échantillons suivie d'une hydrolyse acide puis chromatographie en phase gazeuse).

\subsection{Test de croissance}

II a consisté en la comparaison sur des juvéniles de carpe miroir (Cyprinus carpio) d'un aliment du commerce pour truitelles et d'un aliment à base de zooplancton séché (sécheur cylindre). Les deux aliments présentaient des caractéristiques physiques semblables (granulé de $\varnothing$ $2 \mathrm{~mm}$ ). La formulation de l'aliment expérimental et les compositions analytiques des deux aliments utilisés sont données respectivement dans les Tableaux I et II.

Tableau l: Formule de Paliment expérimental à base de zooplancton ( $(\mathrm{g} / 100 \mathrm{~g}$ de mélange sans eau).

Tablel: $\quad$ Experimental zooplankton feed formula ( $\mathrm{g} / 100 \mathrm{~g}$ of dry mixture).

$\begin{array}{lc}\text { Zooplancton déshydraté } & 64 \\ \text { Blé dur broyé } & 33 \\ \text { Premix vitamines et minéraux } & 2 \\ \text { Alginate de } \mathrm{Na}^{+} & 1\end{array}$

Tableau II: Composition analytique de Paliment expérimental et du témoin ( $g / 100 \mathrm{~g})$.

Tablell: Analytical composition of the experimental zooplankton feed and the commercial feed used as control $(g / 100 \mathrm{~g})$.

\begin{tabular}{lccc}
\hline & $\begin{array}{c}\text { Matière sèche } \\
(\%)\end{array}$ & $\begin{array}{c}\text { Cendres } \\
(\% \text { sur sec })\end{array}$ & $\begin{array}{c}\text { Protéines brutes } \\
\mathrm{N} * 6,25(\% \text { sur sec })\end{array}$ \\
\hline Aliment Zooplancton & 89,8 & 11,9 & 45,2 \\
Aliment témoin & 92,1 & 13,6 & 50,0 \\
\hline
\end{tabular}

L'expérience a été organisée de la façon suivante:

- 5 régimes alimentaires testés en duplicat contenant une part croissante de "protéine zooplancton" (Tableau III) :

- 10 bacs de $1,6 \mathrm{~m}^{3}$ renouvelés en eau à raison d'une fois toutes les 4 heures :

- 170 juvéniles $(0+)$ de carpe miroir (Cyprinus carpio) par bac répartis aléatoirement afin de constituer des lots homogènes (poids individuel moyen de départ: 20 grammes);

- distribution ad libitum des deux aliments en repas alternés (un seul aliment distribué pour un repas donné afin d'éviter le possible choix des poissons si les deux aliments étaient présentés simultanément; voir Tableau III):

- 6 repas par jour à heures fixes, 6 jours par semaine, pour une durée totale de l'expérience de 62 jours. 
Tablesu III : Incorporation crobsante de la protêine zooplancton dans les 5 régimes alimentaires testes sur juréniles de Carpe miroir.

Table III: Increasing incorporation of zooplankton protein in the 5 feeding diets tested on mirror carp juveniles.

\begin{tabular}{ccc}
\hline Régime & $\begin{array}{c}\text { nb. de repas aliment } \\
\text { zooplancton/témoin }\end{array}$ & $\begin{array}{c}\text { protéine zooplancton/protéines totales } \\
(\%)\end{array}$ \\
\hline 0 & $0 / 6$ & 0 (témoin) \\
1 & $1 / 5$ & 13 \\
2 & $2 / 4$ & 27 \\
3 & $3 / 3$ & 40 \\
4 & $4 / 2$ & 57 \\
\hline
\end{tabular}

1.3. Exploltation du zooplancton vivant pour l'élevage larvaire de carpe (Cyprinus carpio) en mode semi-intensif

Ces essais ont été réalisés au centre de recherches du lagunage de Mèze (Hérault), dans deux bassins de $100 \mathrm{~m}^{2}$. Un traitement au Dipterex $\left(1 \mathrm{~g} / \mathrm{m}^{3}\right)$ a été effectué préalablement à l'introduction des larves de carpe afin de favoriser principalement les rotifères et éliminer les formes prédatrices du zooplancton en début d'expérimentation.

Les principaux paramètres de l'expérience ont été les suivants:

- densité d'introduction :

"bassin $1: 37$ larves $/ \mathrm{m}^{2}$

"bassin $2: 96$ larves $/ \mathrm{m}^{2}$,

— durée d'èlevage : 4 semaines,

- renouvellement de l'eau : pour compenser l'évaporation,

- collecte et distribution de zooplancton : selon une succession rotifères-copépodes-daphnies (voir les indications portées au Tableau IV).

Tableau IV: Séquence alimentaire et fréquence de distribution du zooplancton utilisé pour Pélevage larvaire de carpe miroir.

TableIV: Feeding sequence and distribution frequency of live zooplankton for the earty growth of mirror carp fry.

\begin{tabular}{llc}
\hline & Nature du zooplancton & Fréquence de distribution \\
\hline lère semaine & $\begin{array}{c}\text { Rotifères collectés } \\
\text { sur } 71 \mu \mathrm{m}\end{array}$ & 2 fois par jour \\
\hline $\begin{array}{l}\text { 2ème et 3ème } \\
\text { semaines }\end{array}$ & $\begin{array}{l}\text { Copépodes et daphnies } \\
\text { collectès sur } 250 \mu \mathrm{m}\end{array}$ & 1 fois par jour \\
4ème semaine & $\begin{array}{l}\text { Daphnies collectées } \\
\text { sur } 500 \mu \mathrm{m}\end{array}$ & 1 fois par jour \\
\end{tabular}

\section{RÉSULTATS ET DISCUSSION}

\section{Production de zooplancton en étangs de lagunage naturel}

Les valeurs des paramètres de qualité de l'eau ainsi que l'importance de leurs amplitudes journalière et annuelle, caractérisent l'instabilité de ces milieux soumis à un important apport de matières organiques (Tableau $\mathrm{V}$ ).

Les récoltes réalisées au cours de la nuit ont représenté en moyenne $56 \%$ de la biomasse journalière. 
L'intégration des courbes de récolte annuelle (fig. 2) permet le calcul de la biomasse totale de zooplancton potentiellement récoltable. En rapportant ces résultats à la surface des lagunes d'épuration, on obtient en poids frais:

- 1relagune: 6,7 tonnes/ha/an

- $2^{\mathrm{e}}$ lagune: 10,7 tonnes/ha/an

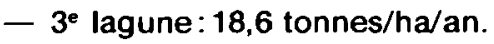
par an.

On retiendra en pratique une valeur moyenne d'environ 10 tonnes de poids frais par hectare et

Si les productions instantanées peuvent être considérablement plus élevées dans les bassins les plus chargés (SL1 > SL2 > SL3 en mars-avril), on obtient les meilleures récoltes annuelles dans les étangs les moins sujets aux variations de charge organique (SL3> SL2) SL1 sur l'année entière). La comparaison avec d'autres installations de lagunage confirme cette analyse.

La production est marquée par une très forte saisonnalité (fig. 2) :

- pic de printemps (Mars-Juin) : $72 \%$ de la récolte annuelle.

- pic hivernal (Novembre-Janvier) : $20 \%$ de la récolte annuelle.

L'identification spécifique a montré uniquement la présence de trois groupes (fig 3 ) :

- cladocères : Daphnia pulex

- copépodes : Cyclops strenuus

- insectes : larves de diptères.

II apparaît très nettement une spécialisation au niveau des organismes présents : daphnies dominantes au printemps, copépodes en hiver.

Tableau V: Lagunage de Réalmont (Tarn) : Valeurs extrêmes relevées pour différents paramétres de qualité de reau.

Table V: $\quad$ Realmont (Tarn, France) domestic sewage treatment ponds. Extrema of various parameter values of water quality.

Paramètres

\section{Mesures}

$\begin{array}{ll}\text { Température } & 3-28,5^{\circ} \mathrm{C} \\ \text { O2 dissous ( } \% \text { saturation) } & 0,5-400 \% \\ \text { pH } & 7,5-10 \\ \text { Transparence (Secchi) } & 10-95 \mathrm{~cm}\end{array}$

Analyses

\section{Ecarts}

$$
\begin{aligned}
& 3-28,5^{\circ} \mathrm{c} \\
& 0,5-400 \% \\
& 7,5-10 \\
& 10-95 \mathrm{~cm}
\end{aligned}
$$

$\begin{array}{ll}\text { Matières en suspension }\left(105^{\circ} \mathrm{C}\right) & 12-128 \mathrm{mg} / 1 \\ \text { OBO5 } & 12-100 \mathrm{mg} / 1 \\ \text { DCO } & 44-560 \mathrm{mg} / 1 \\ \mathrm{~N}-\text { organique } & 0,7-18,6 \mathrm{mg} / 1 \\ \mathrm{~N}-\mathrm{NH4} & 0,3-9,1 \mathrm{mg} / 1 \\ \mathrm{~N}-\mathrm{NO2} & 0,0-1,7 \mathrm{mg} / 1 \\ \mathrm{~N}-\mathrm{NO3} & 0,0-2,7 \mathrm{mg} / 1 \\ \text { Ortho-phosphates } & 2,5-13 \mathrm{mg} / 1\end{array}$




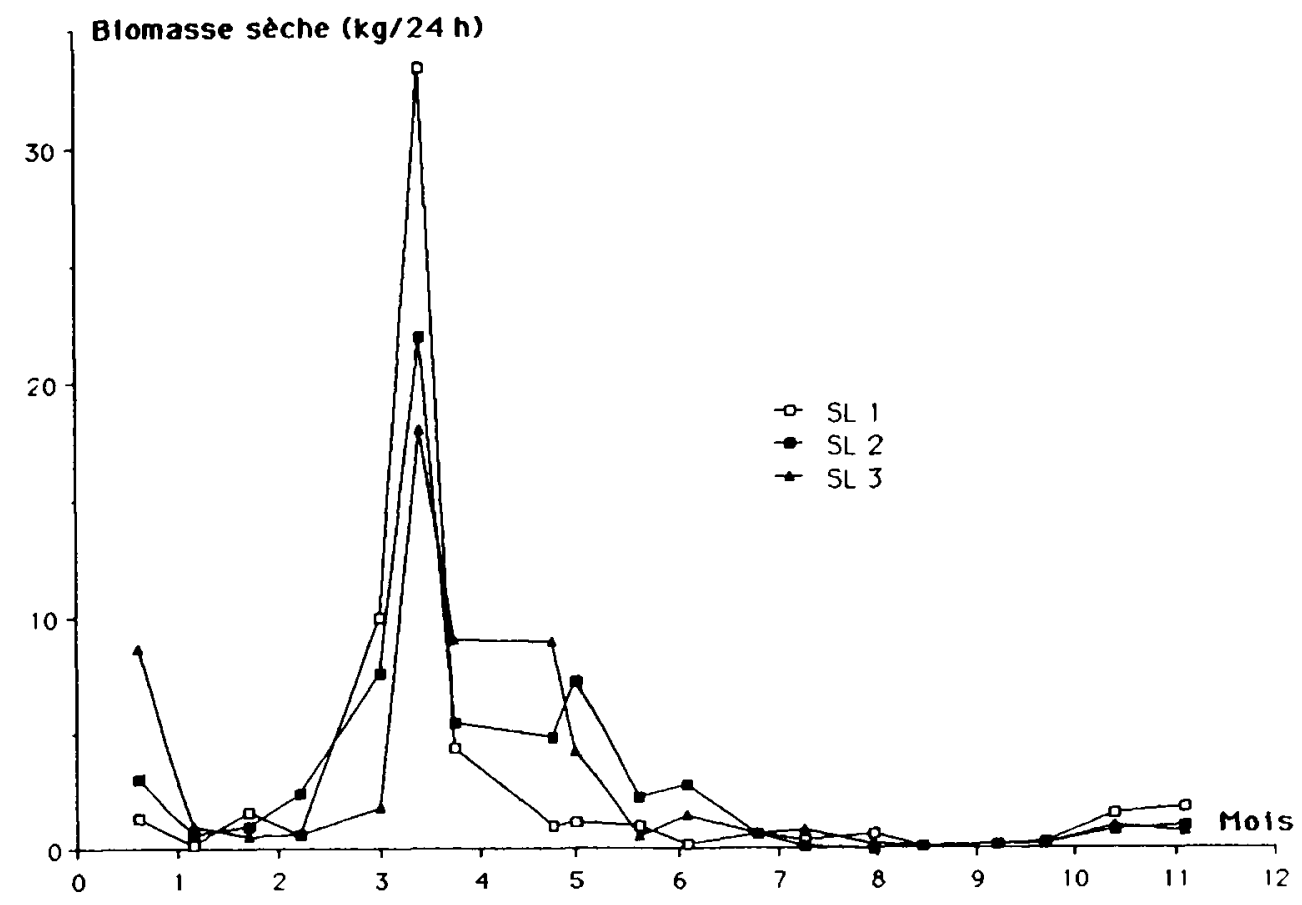

figure 2: Lagunage de Réalmont (Tarn) : évolution des récoltes de zooplancton en sortie de chaque lagune (kg poids sec/24 heures).

figure 2: Realmont (Tarn, France) domestic sewage treatment ponds : evolution of 200plankton harvest in the outflow of each pond (kg of dry biomass/24 hours).

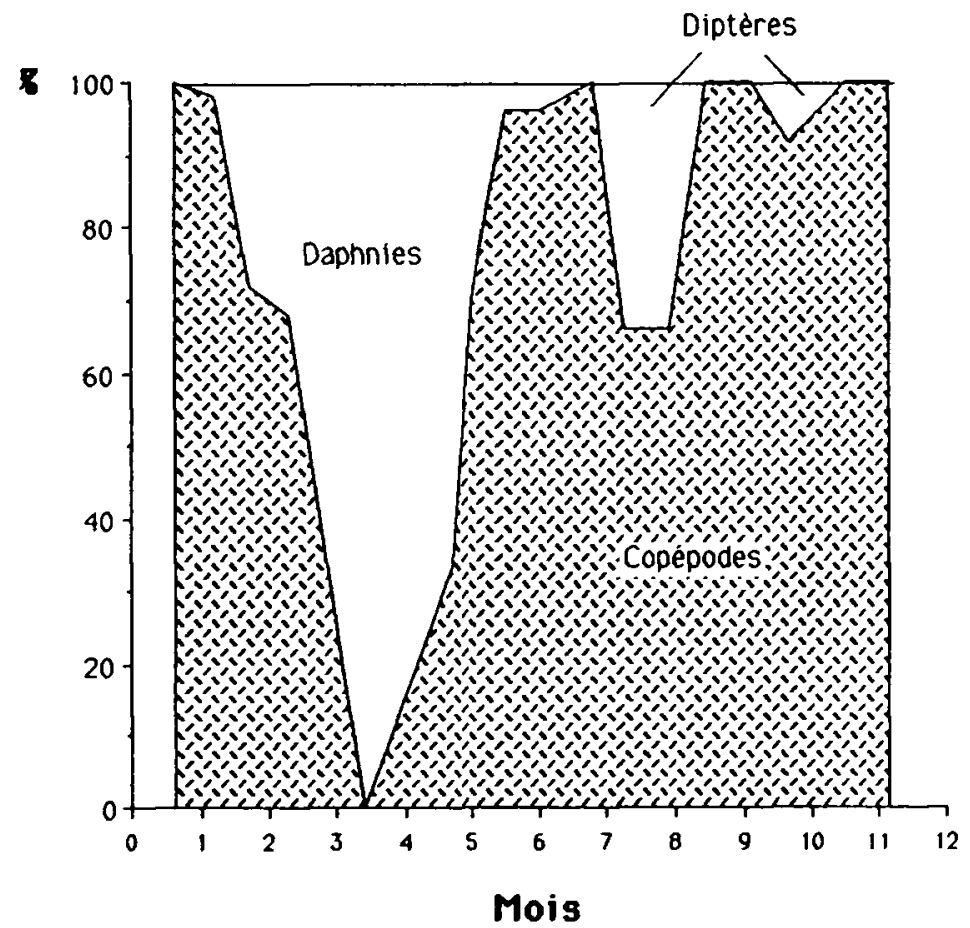

figure 3: $\quad$ Lagunage de Réalmont (Tarn) : abondance relative des trois groupes zooplanctoniques présents (\% du nombre total d'individus).

figure 3: Realmont (Tarn, France) domestic sewage treatment ponds : relative abundance of the three zooplanktonic groups present ( $\%$ of total number of individuals). 
Les fortes biomasses observées et la très faible diversité spécifique du zooplancton quelle que soit la période de l'année, mettent en évidence le caractère hypereutrophe de ces milieux (ANGELI, 1979). La transposition à l'utilisation d'autres fertilisants organiques (lisiers d'élevage par exemple) et à d'autres lieux, n'est pas aisée. En dehors des différences climatiques dont il ne faut pas ignorer l'importance, la nature même de la matière utilisée et son devenir, ne se laissent pas cerner à l'aide de quelques paramètres (TAIGANIDES, 1978). Ainsi, plutỏt que de raisonner en termes de charges d’éléments simples ( $N$, $P$ ) ou de matières oxydables (DB05) à épandre par unité de surface, il nous paraît plus judicieux d'adopter une démarche empirique, consistant à essayer de reproduire la qualité de l'eau obtenue dans nos conditions par un apport progressif du fertilisant disponible. L'état optimal à rechercher se situe à notre avis, dans un intervalle plus étroit autour de la moyenne des valeurs extrẻmes qui ont été exposées au Tableau V.

Les fluctuations mises en évidence tant en ce qui concerne les quantités récoltées que les espèces présentes, constituent un handicap à la valorisation du zooplancton sous forme de proies vivantes en aquaculture, où l'offre doit être étroitement adaptée à la demande des écloseries. Compte tenu de la présence de pics de très forte production notamment au printemps, on peut se demander s'il ne serait pas intéressant d'envisager son utilisation comme base protéique d'aliments composés. Se pose alors de façon plus cruciale encore, le problème de la valeur alimentaire de ce produit.

\section{Valeur alimentaire du zooplancton}

\subsection{Composition analytique}

Les résultats de matière sèche, cendres, protéines brutes sont présentés au Tableau VI en référence à la farine de poisson.

Une analyse de variance de l'ensemble des données a montré que la teneur en matière sèche du zooplancton récolté variait très significativement $(P<0,001)$ au cours de l'année $(6$ à $12 \%$ de M.S.). Ces variations ont été mises en relation avec les fluctuations d'abondance des deux groupes dominants : copépodes et daphnies. Ces dernières présentent une teneur en eau plus élevée.

Tableau VI: Matiere seche, cendres, protêines brules du zooplancton recolté compart a la farine de poisson.

Table VI: Dry matter, ash and crude protein of harvested zocplankton, compared with fish meal.

\begin{tabular}{lccc}
\hline & $\begin{array}{c}\text { Matière sèche } \\
(\%)\end{array}$ & $\begin{array}{c}\text { Cendres } \\
(\% \text { sur sec })\end{array}$ & $\begin{array}{c}\text { Protéines brutes } \\
N * 6,25(\% \text { sur sec })\end{array}$ \\
\hline $\begin{array}{l}\text { Zooplancton } \\
\text { (valeurs moyennes) }\end{array}$ & $10,1 \pm 0,4\left(^{*}\right)$ & $10,8 \pm 2,5(* *)$ & $67,0 \pm 3,6\left(^{* *}\right)$ \\
$\begin{array}{l}\text { Farine de poissons } \\
\text { (d'aprés FAO, 1970) }\end{array}$ & 90,0 & - & 83,3 \\
\hline
\end{tabular}

(*) nombre de données $=114$

(**) nombre de données $=40$

Le taux de protéines brutes $(N * 6,25)$ est en moyenne de $67 \%$ du poids sec. Selon les données publiées par différents auteurs (YURKOWSKI et TABACHEK, 1979; GRABNER et al., 1981 ; WATANABE et al., 1983) les dosages réalisés sur différentes espèces de cladocères et de copépodes du milieu naturel présentent une fourchette de 30 à $75 \%$ de protéines brutes. La validité des comparaisons est toutefois limitée par la variabilité inhérente aux différences d'origine des organismes analysés (DABROWSKI, 1984). 
La comparaison avec les aliments protéiques couramment utilisés en alimentation animale (F.A.O., 1970), classe le zooplancton séché entre la farine de poisson ( $85 \%$ de protéines) et le tourteau de soja ( $50 \%$ de protéines). Cependant, en accord avec certains auteurs (LOVELL et al., 1968; YURKOWSKI et TABACHEK, 1979 ; MATHIAS et al., 1982) il paraît plus juste de corriger le taux de protéines de la teneur en chitine (constituée de glucosamines) ce qui a pour effet de le ramener à $60 \%$ du poids sec.

En tout état de cause, la teneur en protéines du zooplancton suffit à couvrir les besoins quantitatifs de la plupart des poissons étudiés (d'après COWEY, 1979);

- Carpe commune : $38 \%$ de l'aliment sec,

- Truite arc-en-ciel : 40 à $46 \%$ de l'aliment sec,

- Saumon chinook : $40 \%$ de l'aliment sec.

Le Tableau VII montre que les besoins quantitatifs en acides aminés essentiels de la carpe et du saumon chinook sont correctement couverts (d'après COWEY, 1979). Le zooplancton récolté présente de plus un équilibre en acides aminés essentiels qui soutient la comparaison avec la farine de poisson et répond aux exigences de la carpe (fig. 4). DABROWSKI et RUSIECKI (1983) soulignent l'intérêt du zooplancton pour les élevages larvaires et l'alevinage du fait qu'il présente une proportion élevée d'acides aminés libres, plus facilement assimilables. De plus, l'étude de la valeur alimentaire de cet aliment "naturel", peut aider à améliorer la formulation des nourritures composées inertes pour l'élevage larvaire (DABROWSKI, 1984).

Tableau VII : Composition en acides aminés essentiels du zooplancton récolté ( $\mathrm{g} / 100 \mathrm{~g}$ matière sèche) et comparaison avec les besoins de la carpe et du saumon chinook (en g/100 g d'aliment sec - d'après COWEY, 1979).

Table VII : $\quad$ Essential amino-acid composition of harvested 200 plankton ( $\mathrm{g} / 100 \mathrm{~g}$ of dry matter) compared with quantitative requirements of carp and chinook salmon $(\mathrm{g} / 100 \mathrm{~g}$ of dry food - from COWEY, 1979).

\begin{tabular}{lccc}
\hline Acide aminé & Zooplancton $\left(^{*}\right)$ & Carpe & Saumon chinook \\
\hline Arginine & 4,27 & 1,6 & 2,4 \\
Histidine & 1,63 & 0,8 & 0,7 \\
Isoleucine & 2,83 & 0,9 & 0,9 \\
Leucine & 4,79 & 1,3 & 1,6 \\
Lysine & 4,37 & 2,2 & 2,0 \\
Méthionine & 1,33 & $1,2(* *)$ & $1,6(* *)$ \\
Cystine & 0,80 & - & - \\
Phénylalanine & 2,82 & $2,5(* * *)$ & $2,1(* * *)$ \\
Tyrosine & 5,08 & - & - \\
Thréonine & 3,23 & 1,5 & 0,9 \\
Tryptophane & - & 0,3 & 0,2 \\
Valine & 3,71 & 1,4 & 1,3 \\
\hline
\end{tabular}

(*) nombre de données $=12$

(**) en l'absence de Cystine

(***) en l'absence de Tyrosine 


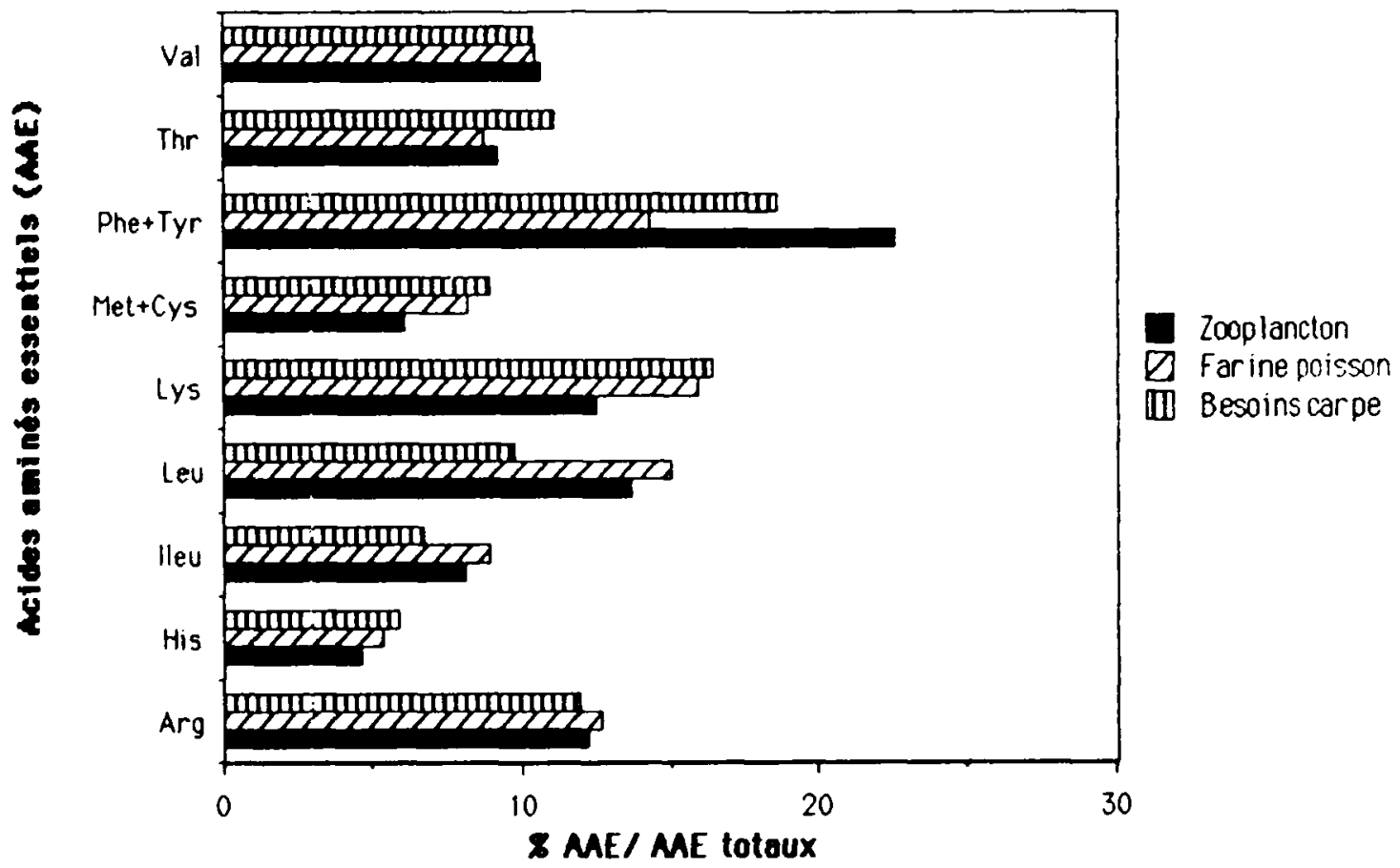

figure 4: Équilibre en acides aminés essentiels (AAE) du zooplancton comparé à la farine de poisson et aux exigences de la carpe (rapport AAE/AAE totaux sauf tryptophane).

figure 4: Essential amino-acid (AAE) pattern in zooplankton, compared with fish meal and carp nutritionnal requirements (ratio AAE/total AAE except tryptophan).

\subsection{Test de croissance}

La comparaison des poids moyens obtenus en fin d'èlevage (Tableau VIII) montre qu'il n'existe pas de différence statistiquement significative entre les régimes 0 et 1 d'une part, 2 et 3 d'autre part.

Tableau VIII: Résultats du test de croissance de juvéniles de carpe miroir avec incorporation croissante de zooplancton dans la ration alimentaire.

Table VIII: Results of the growing test of carp juveniles with increasing incorporation of zooplankton in the diet.

\begin{tabular}{ccccc}
\hline $\begin{array}{c}\text { Régime } \\
\text { Alimentaire }\end{array}$ & \multicolumn{2}{l}{$\begin{array}{c}\text { Poids individuels movens }(g) \\
\text { début } \\
(62 j)\end{array}$} & $\begin{array}{c}\text { Indice } \\
\text { de } \\
\text { consommation } \\
(*)\end{array}$ & $\begin{array}{c}\text { Coefficient } \\
\text { defficacite } \\
\text { protéique } \\
(* *)\end{array}$ \\
\hline 0 & $21,2 \pm 1,1$ & $58,5 \pm 2,4$ & 1,55 & 1,28 \\
1 & $21,4 \pm 1,1$ & $58,0 \pm 2,6$ & 1,65 & 1,22 \\
3 & $20,0 \pm 1,0$ & $52,8 \pm 2,4$ & 1,60 & 1,27 \\
4 & $20,6 \pm 1,0$ & $54,0 \pm 2,1$ & 1,55 & 1,34 \\
& $20,1 \pm 1,1$ & $49,8 \pm 2,3$ & 1,76 & 1,20
\end{tabular}

$\left.{ }^{*}\right) \mathrm{kg}$ daliment distribue $/ \mathrm{kg}$ de polsson prodult

(*) kg poisson produit/kg de protéines contenues dans la ration distribuee 
Afin de corriger deux biais introduits dans l'expérience (appétence plus faible (1) constatée pour l'aliment zooplancton et taux de protéines inférieur de $5 \%$ par rapport au témoin) il paraît légitime de se référer aux indices relatifs (Tableau VIII) : indice de consommation (IC = kg d'aliment distribué/kg de poisson produit) et coefficient d'efficacité protéique (CEP $=\mathrm{kg}$ de poisson produit/kg de protéines contenues dans la ration distribuée). Le régime permettant d'obtenir le meilleur compromis entre efficacité et performance absolue paraît être le régime 3 . Celui-ci procure un indice de consommation minimum et un coefficient d'efficacité protéique maximum, en ne diminuant le poids moyen en fin d'expérience que de $7,7 \%$ par rapport au témoin. Son utilisation aura permis l'épargne de $\mathbf{4 0} \%$ de protéines conventionnelles par la protéine zooplancton (cf. Tableau III).

\subsection{Valorisation comme protèine alternative}

Ainsi la bonne teneur en protéines et en acides aminés du zooplancton permet d'envisager en principe son utilisation comme protéine alternative à la farine de poissons pour la fabrication d'aliments composés. Cependant, du fait de sa très forte teneur en eau (environ $90 \%$ ) cette protéine est très "diluée": $6 \%$ du procuit frais contre à peu près $18 \%$ pour la viande de bceuf et le poisson (F.A.O., 1970). Ceci constitue une limite évidente à sa valorisation comme ingrédient d'aliments composés en impliquant une étape de déshydratation préalable et en diminuant d'autant sa quantité disponible "utile" (1 tonne/ha/an de matière sèche). Ce facteur paraît, en l'état actuel, rédhibitoire à toute industrialisation. En effet, si l'on souhaitait substituer significativement la "protèine zooplancton" à la farine de poissons dans l'élaboration des quelques $\mathbf{5 0 . 0 0 0}$ tonnes d'aliments consommés annuellement par la production de truites françaises cela nécessiterait pour un simple taux d'incorporation de $10 \%, 5.000$ tonnes de zooplancton sec, soit 5.000 hectares de lagunes d'épuration... soit une capacité de traitement correspondant environ au dixième de la population française ( 1 ha de lagunage pour 1.000 habitants traitès). On est bien loin de disposer d'une superficie aussi imposante de lagunes d'épuration, et l'on n'en disposera vraisemblablement jamais!

Ceci n'empêche pas une valorisation à l'échelle artisanale sous forme d'aliment composé sec ou semi-humide, ou encore une commercialisation de zooplancton congelé destiné à des créneaux spécifiques (aquariophilie, écloseries), comme cela se pratique à Mèze depuis plusieurs années.

3. Exploitation du zooplancton vivant pour l'élevage larvaire de carpe (Cyprinus carpio) en mode semi-intensif

Les performances obtenues en 4 semaines d'élevage sont :

- $70 \%$ de survie et $1,95 \mathrm{~g}$ de poids individuel moyen pour le bassin 1 .

— près de $99 \%$ de survie et $0,67 \mathrm{~g}$ pour le bassin 2 (Tableau IX).

La température de l'eau a varié entre 20 et $30^{\circ} \mathrm{C}$.

Les quantités de zooplancton distribué ont représenté $46 \mathrm{~kg}$ pour le bassin 1 et $84 \mathrm{~kg}$ pour le bassin 2 (poids frais égoutté).

Tableau IX: Survie et poids moyens d'alevins de carpe miroir èlevés de 0 à 4 semaines à l'aide de zooplanctón collecté en lagune d'épuration.

Table IX: $\quad$ Survival and average weight of mirror carp fry reared from 0 to 4 weeks, with live zooplankton collected in waste water ponds.

\begin{tabular}{cccccc}
\hline $\begin{array}{l}N^{*} \\
\text { bassin }\end{array}$ & $\begin{array}{c}\text { no larves } \\
\text { introduites }\end{array}$ & $\begin{array}{c}\text { no dalevins } \\
\text { produits }\end{array}$ & $\begin{array}{c}\text { taux de } \\
\text { survie (\%) }\end{array}$ & $\begin{array}{c}\text { Poids moven }(g) \\
\text { larves }\end{array}$ & \begin{tabular}{c} 
alevins \\
\hline 1
\end{tabular} \\
\hline 3700 & 2600 & 70,2 & 0,001 & $1,95 \pm 0,19$ \\
2 & 9600 & 9500 & 98,9 & 0,001 & $0,67 \pm 0,07$
\end{tabular}

(1) Sans doute due à la formulation rudimentaire de l'aliment experimental (qui ne comportait en particulier aucune adjonction de lipides) par rapport au temoin. 
Ces résultats sont excellents si on les compare à ceux obtenus, pour la même durée d'élevage. dans les carpicultures réputées performantes : en Hongrie avec contrỏle de la biocénose planctonique, le taux de survie à 4 semaines s'établit entre $\mathbf{4 0}$ et $60 \%$ de l'effectif introduit pour un poids individuel moyen d'environ 1 gramme (MARCEL, 1986). De mêmeà FLEVO-LELYSTAD (Pays-Bas), après élevage en eau thermorégulée à $24^{\circ} \mathrm{C}$ les poids moyens sont de l'ordre de 0,8 à 1 gramme (GUERRIN, 1983). En Camargue, par des méthodes plus traditionnelles les poids moyens à 4 semaines allaient de 0,2 à 0,5 gramme (RICARD, 1981).

La croissance pondérale individuelle a été trois fois plus élevée dans le bassin 1 en comparaison du bassin 2, pour une densité de larves introduites trois fois plus faible. Si l'on prend en compte la quantité de zooplancton distribué rapportée au volume d'élevage, à l'effectif des larves introduites, et au nombre d'alevins produits (Tableau $X$ ), on constate les faits suivants :

- dans le bassin 1, la quantité de nourriture distribuée per capita a été très nettement supérieure à celle distribuée aux alevins du bassin 2 ;

- dans le bassin 2, la densité cumulée de zooplancton dans le volume d’élevage a été près de deux fois plus importante en comparaison du bassin 1.

Or, les résultats de survie sont meilleurs en 2 qu'en 1, et la croissance meilleure en 1 qu'en 2.

De plus en termes d'efficacité alimentaire, les indices de consommation obtenus établissent à environ $9 \mathrm{~g}$ de zooplancton distribué/g d'alevins produits pour le bassin 1 (faible densité) et à 13 pour le bassin 2 (forte densité). Il apparaît donc une meilleure utilisation de la nourriture disponible à faible densité de larves introduites et de proies distribuées.

Tableau X: Premier alevinage de carpe miroir : quantité totale de zooplancton distribué (g poids frais) rapportée au volume óélevage, a leffectif de larves introduites et a leffectif d'alevins obtenus à 4 semaines.

Table X : $\quad$ Early growth of mirror carp : total amount of distributed live zooplankton ( $\mathrm{g}$ of fresh weight) per water volume, per initial number of larvae, and per number of fry at 4 weeks.

\begin{tabular}{|c|c|c|c|}
\hline \multirow{2}{*}{$\begin{array}{l}\mathrm{N}^{\bullet} \\
\text { bassin }\end{array}$} & \multicolumn{3}{|c|}{ quantité de zooplancton distribué } \\
\hline & $\mathrm{g} / \mathrm{m} 3 \mathrm{~d}$ eau & $\mathrm{g} /$ larve introduite & $g /$ alevin produit \\
\hline 1 & 512 & 12,4 & 17,7 \\
\hline 2 & 936 & 8,7 & 8,8 \\
\hline
\end{tabular}

Or, les résultats de survie sont meilleurs en 2 qu'en 1, et la croissance meilleure en 1 qu'en 2.

De plus en termes d'efficacité alimentaire, les indices de consommation obtenus s'établissent à environ $9 \mathrm{~g}$ de zooplancton distribué/g d'alevins produits pour le bassin 1 (faible densité) et à 13 pour le bassin 2 (forte densité). Il apparaît donc une meilleure utilisation de la nourriture disponible à faible densité de larves introduites et de proies distribuées.

Avec les réserves d'interprétation qu'impose l'absence de réplicats dans cette expé rience, nous en concluons que :

- une densité élevée de proies dans le milieu d'élevage favorise la survie au stade critique où les larves ont un comportement statique ( $1^{\text {re }}$ semaine d'élevage) ;

- une distribution proportionnée à l'effectif d'alevins est ensuite nécessaire $\left(2^{\circ}\right.$ à $4^{*}$ semaine) afin d'optimiser la croissance et l'efficacité alimentaire, dès lors que les animaux ont acquis un comportement de recherche active des proies.

C'est effectivement le principe sur lequel est basée la production d'alevins de Cyprinidés à l'écloserie-nourricerie de FLEVO-LELYSTAD au Pays-Bas (pour plus de détails voir GUERRIN, 1983). L'alevinage y est pratiqué en eau thermorégulée $\left(24^{\circ} \mathrm{C}\right)$ et comprend deux phases :

- $1^{\text {re }}$ semaine : en aquariums à très haute densité (180 à 250 larves/litre) :

$-2^{e}$ à $4^{e}$ semaine : en bacs de $4 \mathrm{~m}^{2}$ à raison de 7.500 à 15.000 alevins par mètre carré.

Le zooplancton est distribué la première semaine de façon à maintenir une densité maximale de proies vivantes dans les aquariums. Les quantités déversées sont ensuite proportionnées au stock d'alevins de la $2^{e}$ à la $4^{e}$ semaine. Dans ces conditions, la durée d'élevage de carpes de $1 \mathrm{~kg}$ a été ramenée de 3 étés à 2 étés. Un schéma similaire est appliqué aux autres cyprinidés, en particulier la carpe herbivore (Ctenopharyngodon idella). 
Ainsi, les apports de zooplancton trié, vivant, permettent d'obtenir en conditions semiintensive à intensive, d'excellents résultats, en termes de survie et de croissance, dans l'alevinage de la carpe et d'autres Cyprinidés. La forte part de main-d'œuvre, impliquée dans les opérations de collecte et de distribution du plancton, pose néanmoins un problème de coût dès que l'objectif porte sur la production d'un grand nombre d'alevins. Augmenter la densité de post-larves et limiter cette pratique aux deux premières semaines de la vie trophique permet de réduire ces inconvénients.

Malgré les difficultés technologiques créées par les fluctuations de la densité du zooplancton dans l'eau des lagunes d'épuration, la mise au point d'un procédé automatisé de récolte-tridistribution paraît souhaitable. Le prototype de récolteur-trieur décrit par BARNABÉ (1984) est à ce titre d'un grand intérêt.

II est d'autre part essentiel pour la réussite de ce type d'élevage, de prendre en compte le comportement trophique des larves (KENTOURI, 1985).

\section{CONCLUSION}

Moyennant quelques améliorations, la collecte de zooplancton en étangs fortement fertilisés paraît toujours digne d'intérêt au regard des difficultès d'ordre technico-économique rencontrées dans la production de proies en milieu contrôlé. II semble que ces deux filières puissent venir en complément l'une de l'autre, en attendant la mise au point d'aliments inertes et de systèmes fiables qui viennent définitivement régler les problèmes de l'alimentation larvaire en pisciculture.

\section{REMERCIEMENTS}

- au Conseil Régional Miđi-Pyrénées, au Conseil Général du Tarn, à l'Agence de l'eau AdourGaronne qui ont subventionné l'étude menée sur le lagunage de Réalmont ;

- à la municipalité de Réalmont et au Centre de recherches du lagunage de Mèze qui ont mis à disposition leurs installations ;

- à MM. J.M. Fesquet, A. Siré, H. Jogand, A. Rantetondok, stagiaires au CEMAGREF pour leur participation active à ces études ;

- à M. P. Luquet, INRA, pour les conseils donnés dans l'étude de la valeur alimentaire du zooplancton. 


\section{RÉFÉRENCES}

ANGELI N., 1979. Relations entre le plancton et la qualité de l'eau : incidence des polluants sur le profil biologique de quelques canaux et étude comparative des interactions plancton/autoépuration en bassins expérimentaux de lagunage. Thèse d'État, Univ. sci. et techn. de Lille, 277 p. + annexes 144 p.

BARNABÉ G., 1979. Utilisation des chaînes alimentaires naturelles et du recyclage des eaux usées dans la production à grande échelle de juvéniles pour l'Aquaculture. Publ. Sci. Tech. CNEXO: Actes Colloq., 7 p. 221-238.

BARNABE G., 1984. Utilisation de plancton collecté pour l'élevage de masse de poissons marins. In : G. Barnabé et R. Billard Ed., l'Aquaculture du Bar et des Sparidés, INRA publ., Paris, p. 185-207.

CHARLON N. et BERGOT P., 1984. Rearing system for feeding fish larvae on dry diets; trial with carp (Cyprinus carpio L.) larvae. Aquaculture, 41 (1984), p. 1-9.

COWEY C.B., 1979. Protein and amino-acid requirements of finfish. In : Proc. world sympostum on finfish nutrition and fishfeed technology, Hamburg 20-23 june 1978, vol. 1, p. 3-16.

DABROWSKI K., 1984. The feeding of fish larvae : present "state of the art" and perspectives. Reprod. Nutr. Develop., 1984, 24 (6), p. 807-833.

DABROWSKI K. et RUSIECKI M., 1983. Content of total and free amino-acids in zooplanktonic food of fish larvae. Aquaculture, 30 (1983), p. 31-42.

F.A.O., 1970. Teneur des aliments en acides aminés et données biologiques sur les protéines. Études de nutrition de la F.A.O., $n^{\circ} 24$, Rome.

GRABNER M., WIESER W. et LACKNER R., 1981. The suitability of frozen and freeze dried zooplankton as food for fish larvae : a biochemical test program. A quaculture, 26 (1981), p. 85-94.

GUERRIN F., 1981. Le lagunage naturel des eaux usées des collectivités rurales ; expérience acquise dans le bassin Adour-Garonne. Rapport Agence de Bassin Adour-Garonne, fascicule 2, $55 \mathrm{p}$.

GUERRIN F., 1983. Elevage intensif de brochetons et alevins de repeuplement aux Pays-Bas. Rapport de mission, CEMAGREF, Montpellier, $31 \mathrm{p}$.

KENTOURI M., 1985. Comportement larvaire de 4 sparidés méditerranéens en élevage. Thèse d'État, USTL, Montpellier, Mai 1985, 492 p.

LOVELL R.P., LAFLEUR J.R. et HOSKINS F.H., 1968. Nutritionnal value of freshwater crayfish waste meal. J. agr. food chem., vol 16, no 2, mar.-apr. 1968, p. 204-207.

MARCEL J., 1986. La pisciculture en étang. In : Barnabé G., Aquaculture, vol. 2, p. 571-608.

MATHIAS J.A., MARTIN J., YURKOWSKI M., LARK J.G.I., PAPST M. et TABACHEK J.L., 1982. Harvest and nutritionnal quality of Gammarus lacustris for trout culture. Trans. Am. fish. soc., 111, 83-89.

RICARD J.M., 1981. Developpement des techniques de contrôle de la reproduction de la carpe commune en écloserie. Mémoire de fin d'études ENSAA Dijon/CEMAGREF Montpellier, 149 p.

TAIGANIDES E.P., 1978. Principles and techniques of animal waste management and utilization. FAO soils bull. (1978), 36, p. 341-362.

TAMAS G. et HORVATH L., 1976. Growth of cyprinids under optimal zooplankton conditions. Bamidgeh, vol. 28 (1976), $n^{\circ} 3$, p. $50-56$.

WATANABE T., KITAJIMA C. et FUJITAS S., 1983. Nutritionnal values of live organisms used in Japan for mass propagation of fish : a review. Aquaculture, 34 (1983), p. 115-143.

YURKOWSKI M. et TABACHEK J.L., 1979. Proximate and amino-acid composition of some natural fish foods. Proc. world symposium on finfish nutrition and fishfeed technology, Hamburg 20-23 june 1978, vol. 1, p. 435-448. 